\title{
A Ictiofauna da Bacia do Rio Paraíba do Sul. Biodiversidade e Padrões Biogeográficos.
}

\author{
Carlos Roberto S. Fontenelle Bizerril \\ Universidade do Rio de Janeiro (UNI-RIO), Laboratório de Avaliação Ambiental. Consultor da Cooperação \\ Brasil/França para o Projeto Paraíba do Sul. Endereço para correspondência: rua Roquete Pinto, no59, apto. 202, \\ Urca,Rio de Janeiro, RJ, Brasil. CEP: 22291-210.
}

\begin{abstract}
Fish fauna taxonomic composition and biogeographic informations of the Paraíba do Sul river basin are presented. Samples were taken in 75 units between January/1995 and June/1998. Species were identified and classified according its distributional pattern. Biogeographic analyses were conduced using macro, meso and micro scales. A total of 167 species were sampled, being 37 marine species. Among the marine taxa, Awaous tajasica and Oosthetus lineatus seems to spend their whole life histories in the freshwater environments. Sixteen species were introduced in the basin. Most of the native taxa inhabit the rivers'storage zone, mainly in pools. Faunistic similarities between the Paraiba do Sul river basin and other Brazilian rivers suggest that the environment studied were formed by the capture of portions of the rivers Grande and Tietê (Paraná river basin) and of small streams that nowadays flows directly from the Serra dos Orgãos hills to the Atlantic ocean. Five main endemism areas were recognised in the studied basin. Another five subareas presented low levels of biotic integrity, resulting in a visible process of extinction.
\end{abstract}

Key words: Biodiversity, Paraíba do Sul river basin, freshwater fishes, southeastern Brazil.

\section{INTRODUÇÃO}

Bacias hidrográficas localizadas na província biogeográfica do sudeste brasileiro (sensu Bizerril, 1994) caracterizam-se por exibirem pequenas dimensões, usualmente com baixa complexidade de sua rede fluvial. Este aspecto, associado à ocorrência natural de processos estocásticos de alteração ambiental, determinam a dinâmica evolutiva das populações que integram suas ictiocenoses.

Estudos realizados (Bizerril, 1995a; Bizerril et al., 1996) vêm indicando um processo evolutivo regional marcado pelo predomínio de eventos de extinção sobre a ocorrência de especiação. Neste sentido, o manejo das bacias hidrográficas do sudeste brasileiro mostra-se particularmente delicado, visto que a baixa capacidade de tamponamento gera uma alta susceptibilidade de ocorrência de perdas bióticas em decorrência de impactos antrópicos, magnificando e acelerando um processo inerente da área.

No entanto, como já é plenamente sabido, o quadro atual de uso do espaço da região aponta para um sentido oposto ao recomendado, já se evidenciando na área inúmeros casos de alterações críticas sobre os ecossistemas locais (Drumond, 1988). A implantação de um modelo de análise integrada de bacias hidrográficas como diretriz para o manejo de tais elementos da paisagem é a meta dos comitês de bacias, alguns em fase de formação e outros já legalmente consolidados.

A bacia do rio Paraíba do Sul, o maior sistema hidrográfico da região biogeográfica do sudeste brasileiro e o sistema com maior importância econômica e estratégia desta unidade geográfica, é uma das bacias brasileiras na qual se estuda, mediante a Cooperação Brasil-França, o modelo de implantação e atuação de uma agência de bacia. Neste sentido, foram reunidos, durante nove anos de projetos, informações sobre elementos estratégicos relacionados à bacia em questão, abordando aspectos socio-econômicos, físicos e bióticos.

No componente síntese "meio biótico", a ictiofauna, estudada pelo autor a partir de 1995, foi o grupo escolhido, por seu alto conteúdo 
sistêmico, para a avaliação biótica dos ecossistemas aquáticos que compõem a bacia do Paraíba do Sul.

Dentro de uma perspectiva histórica, este conjunto biótico da bacia do rio Paraíba do Sul começou a ser estudado a partir da descrição, ainda nos séculos XVIII e XIX, de espécies coligidas no sistema. No plano de taxonomia básica, trabalhos mais recentes, como os de Langeani (1990), Caramaschi \& Caramaschi (1991), Costa (1992), Mazzoni et al. (1993), Bizerril (1995b) e Bockmann et al. (1996) apresentaram novos taxa, bem como elucidaram alguns aspectos da taxonomia de grupos já catalogados.

Levantamentos faunísticos da bacia são menos abundantes e não abrangem toda a bacia. Podese considerar como o primeiro estudo geral da ictiofauna local, o trabalho de Miranda-Ribeiro (1902), que apresentou algumas observações sobre 8 espécies de peixes do rio Pomba, um tributário do sistema do Paraíba do Sul.

Dos estudos mais recentes, destacam-se os trabalhos de Britski (1972) que, ao descrever a ictiofauna do Estado de São Paulo, listou as espécies do Paraíba do Sul, com especial ênfase às presentes em território paulista, de Nunann $e t$ al. (1983), que apresentaram um levantamento da ictiofauna do Paraíba do Sul entre a UHE de Funil e a cidade de Barra do Pirai, de Araújo (1983), que ao coordenar o "Projeto de Biotetecção de Tóxicos em Sistemas Fluviais de Utilização em Captação de Água em Sistemas Públicos de Abastecimento", relacionou as espécies da bacia, da ENGEVIX/URFJ (1991), que enfocou o trecho entre Três Rios e Campos dos Goytacazes, de Araújo (1985; 1996), que tratou da composição e da estrutura da comunidade de peixes do médio e baixo Paraíba do Sul, e de Espirito-Santo et al. (1997), que relatam a ocorrência de 28 espécies de peixes em Pindamonhangaba, SP.

O presente trabalho, essencialmente descritivo, ao mesmo tempo que analisa criticamente a base dados disponível, sintetiza resultados alcançados nos estudos da Cooperação Brasil-França que se referem à composição taxonômica das comunidades de peixes da região e a aspectos biogeográficos, identificando áreas que atualmente mostram maior prioridade para a manutenção deste segmento da biota local.

\section{METODOLOGIA}

A bacia do rio Paraíba do Sul abrange uma área de aproximadamente $57.000 \mathrm{~km}^{2}$, compreendida entre os paralelos $20^{\circ} 26^{\prime}$ e $23^{\circ} 38^{\prime}$ sul e os meridianos $41^{\circ}$ e $46^{\circ} 30^{\prime}$ oeste. Ao norte seu divisor de águas se faz entre os rios Grande (bacia do Paraná) e Doce (sistema do leste brasileiro) por intermédio da Serra da Mantiqueira. Ao sul, a Serra do Mar separa esta bacia de diversos pequenos rios que fluem diretamente para o Oceano Atlântico.

Na região leste o isolamento da bacia do Paraíba se faz por meio de relevos montanhosos localizados entre a Serra da Mantiqueira e a Serra do Mar que separa este sistema do rio Itabapoana. A oeste o Paraíba do Sul apresenta divisores de água com o rio Tietê (bacia do Paraná), do qual é separado por meio de diversas ramificações dos maciços da Serra do Mar e da Serra da Mantiqueira.

O rio Paraíba do Sul é formado pela união do rio Paraitinga com o Paraibuna. A direção do canal principal sofre sensíveis variações ao longo de seu traçado, que corre inicialmente na direção sul-oeste, guinando abruptamente à direita, tomando direção noroeste, a qual é mantida na maior parte do percurso médio, exceto em um curto trecho compreendido entre Cachoeira Paulista e Barra do Piraí, onde o rio corre para leste.

Nos últimos $80 \mathrm{~km}$ de curso inferior, o rio Paraíba do Sul, após receber carga do rio Pomba, deixa as formações cristalinas e segue, em inúmeros meandros, para leste, atingindo o oceano Atlântico.

Ao longo do seu traçado e do curso da rede de tributários, a bacia do rio Paraíba do Sul encontra-se representada nos estados de São Paulo, Rio de Janeiro e de Minas Gerais. Destes, o Estado do Rio de Janeiro é o que exibe maior área drenada pelo sistema, com 37,9\% da área total. Seguem-se os estados de São Paulo $(37,7 \%)$ e Minas Gerais $(24,4 \%)$ (Costa, 1994). 
Integrando o perfil com o aspecto da paisagem dos diferentes setores da bacia, avaliado em campo e/ou em cartas topográficas do IBGE (Escalas 1:250.000 e 1.50.000), pode-se reconhecer a existência de domínios fluviais (i.e. unidades ambientais). Bizerril (1996) reconheceu sete domínios, assim denominados:DI - Domínio das Serras e do Planalto, DII - Domínio dos meandros com lagoas marginais, DIII - Domínio de meandros com condicionamento estrutural, DIV - Domínio das corredeiras, DV- Domínio das ilhas fluviais, DVI - Domínio dos depósitos fluviais e DVII Domínio das lagoas, adotando uma terminologia para a denominação dos domínios que expressa a dominância de determinados elementos da paisagem. A localização de cada domínio dentro do perfil longitudinal do rio Paraíba do Sul encontra-se na Fig. 1.

O presente estudo teve início com a ordenação dos dados secundários e avaliação crítica dos mesmos revendo, a partir do exame de material coletado na bacia, as designações específicas conferidas aos diferentes grupos ícticos pelos autores consultados.

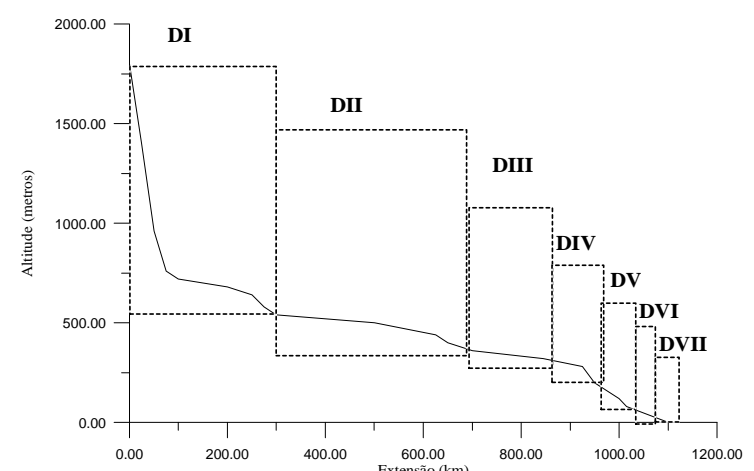

Fig. 1. Domínios geoambientais do rio Paraíba do Sul.

Posteriormente, foram realizadas campanhas, entre janeiro de 1995 e junho de1998 em um total de 75 áreas de amostragem (Fig. 2), com vistas a ampliar e consolidar a base de dados disponível sobre a ictiofauna local, observandose o uso de recursos tróficos e espaciais pelos taxa da região. Todo o material coletado encontra-se depositado no Laboratório de Avaliação Ambiental da Universidade do Rio de Janeiro (UNI-RIO).
Considerando não ser parte do escopo do presente estudo realizar revisão taxonômica dos grupos de peixes da bacia do rio Paraíba do Sul, uma atividade que vem sendo desenvolvida por outros pesquisadores, alguns dos grupos locais foram identificados apenas ao nível de gênero. Para outros, adotou-se a nomenclatura atualmente em uso, destacando, mediante o emprego de cf. (a conferir) e aff. (afim de) a necessidade de revisão do status taxonômico.

No caso específico das espécies introduzidas, foi também adotada a classificação ecológica sugerida por Lodeg (1993), considerando os termos "colonizador", para designar espécies que tenham sido introduzidas em determinado ambiente, e "invasor" no caso de espécies que possuam populações aparentemente autosustentáveis.

Para a caracterização complementar dos grupos nativos relacionados foi considerado o tipo de ambiente preferencialmente ocupado por cada espécie, considerando a variação ambiental registrada.

Quanto a este aspecto, a descrição foi realizada sintetizando os dados de campo em três escalas de detalhamento, correspondentes as aproximações macroespacial, mesoespacial e microespacial.

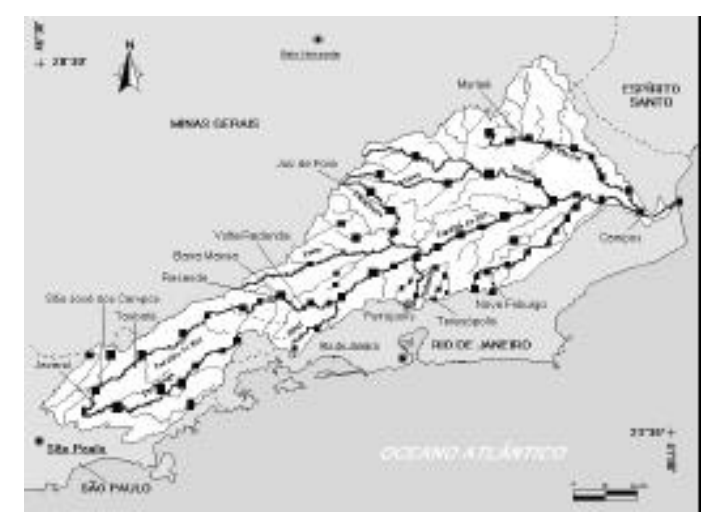

Fig. 2. Localização das áreas de amostragem do presente estudo (Uma área pode representar mais de um ponto de coleta)

Para descrever os padrões de distribuição macroespaciais da ictiofauna foi seguida a compartimentação geoambiental do rio Paraíba do Sul nos sete domínios (ver Fig.1), associando aos mesmos a rede de drenagem adjacente. 
A aproximação mesoespacial objetivou descrever o uso do espaço considerando a perspectiva longitudinal dos sistemas lóticos como um todo. Para tanto, foi adotada a divisão funcional dos hidrossistemas proposta por Petts \& Amoros (1997), na qual o sistema fluvial pode ser compartimentado nas zonas de fonte ou produção (production zone), na qual predominam os processos erosivos estabelecidos sobre unidades de pequena hierarquia fluvial, de transporte ou transferência (transfer zone), correspondendo ao setor no qual o rio, já dotado de maiores dimensões, apresenta um regime funcional baseado no predomínio de processos de transporte sobre a atividade erosiva como um todo, e de armazenamento (storage zone), onde se verifica a dominância da atividade de deposição.

Em termos microespaciais, a compartimentação se deu para cada uma das zonas funcionais, objetivando ilustrar a variação ambiental geral de cada setor. Assim, foi adotada a divisão apresentada na Fig. 3, a qual representa o arranjo dominante observado ao longo dos trabalhos de campo.

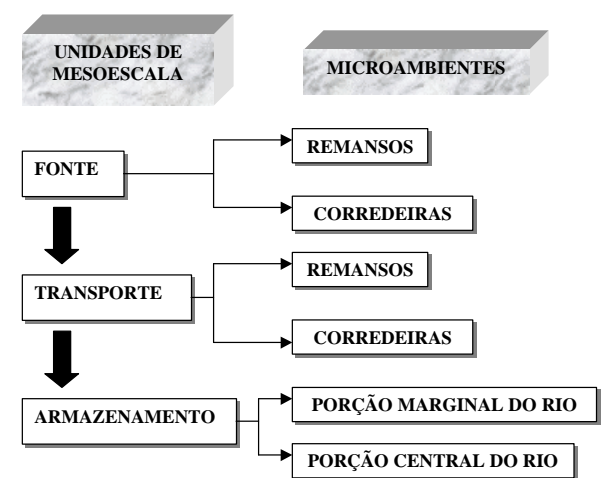

Fig. 3. Divisão adotada para a análise

\section{RESULTADOS E DISCUSSÃO}

Como resultado do levantamento ictiofaunístico constatou-se que a bacia do rio Paraíba do Sul conta com 167 espécies de peixes. Alguns estudos, se confirmados, podem vir a elevar o número de espécies da bacia. Como exemplo, pode-se mencionar, além das análise de revisão taxonômica em curso, o trabalho de Suppa \& Buckup (1997), no qual os autores reconhecem, em resumo de congresso, 8 espécies de Rineloricaria na bacia, enquanto no presente trabalho consideramos apenas a existência de 4 morfoespécies

Os taxa inventariados na bacia podem ser ordenados em: espécies de água doce nativas (68,5\% do total amostrado), espécies marinhas (22\%) e espécies dulcícolas introduzidas $(9,5 \%)$.

Os grupos marinhos relacionados na Tabela I são, em sua maioria, comuns à sistemas lagunares e à desembocadura de rios (cf. Andreata et al., 1990; Bizerril, 1995b, Bizerril et $a l .$, 1995), denotando alta valência às variações de salinidade.

Tais grupos tendem a exibir distribuição limitada ao ultimo domínio ambiental da bacia (i.e., domínio das lagoas marginais - DVII), ocorrendo na área próxima à foz do rio Paraíba. Algumas espécies, contudo, apresentam uma distribuição mais ampla (Fig. 4)

Destas, O. lineatus e A.tajasica embora possuam baixa capacidade de dispersão ativa, devido ao pequeno porte $(<15 \mathrm{~cm})$ foram coletados em diferentes estádios de desenvolvimento no interior da bacia, incluindo as fases de juvenil, imaturo e de adulto com gonadas bem desenvolvidos, o que sugere se tratar de taxa que fecham o ciclo vital no interior da bacia.

Platanichthys platana, embora não tenha sido registrada após a desembocadura do rio Muriaé, ocupa diversas lagoas marginais do rio Paraíba do Sul (i.e., Lagoa da Cataia, Lagoa do Jacu), ambientes caracterizados pelas condições eminentemente oligohalinas de suas águas. Este padrão foi igualmente observado por ENGEVIX/UFRJ (1991).

Um conjunto ictiofaunístico recente na bacia é aquele representado pelas espécies exóticas. Atualmente, dezesseis espécies de peixes alóctones, procedentes da América do Norte, América Central Europa, África, Ásia e de outros rios sul americanos podem ser registradas no Paraíba do Sul. A classificação das espécies nestas categorias propostas por Lodge (1993) em cada domínio geoambiental do Paraíba do Sul é apresentada na Tabela II. 
Tabela I. Espécies marinhas da bacia do rio Paraíba do Sul.

\begin{tabular}{||l||l||}
\hline ANGUILIFORMES & SCIAENIDAE \\
OPHICHTHYIDAE & Micropogonias furnieri \\
Myriophis punctatus & Bairdiella ronchus \\
CLUPEIFORMES & CARANGIDAE \\
CLUPEIDAE & Caranx bartholomaei \\
Platanichthys platana & C. latus \\
ENGRAULIDIDAE & C. lugubris \\
Anchoa januaria & Oligoplites saurus \\
Anchovia clupeoides & GERREIDAE \\
Anchoviella lepidentostole & Gerres aprion \\
SILURIFORMES & Diapterus rhombeus \\
ARIIDAE & D. olisthostomus \\
Netuma barba & GOBIIDAE \\
Cathrops spixii & Awous tajasica \\
Genidens genidens & Bathygobius soporator \\
Sciadeichthys luniscutis & Gobionellus oceanicus \\
ATHERINIFORMES & Gobionellus sp. \\
ATHERINIDAE & ELEOTRIDIDAE \\
Xenomelaniris brasiliensis & Eleotris pisonis \\
BELONIFORMES & Dormitator maculatus \\
BELONIDAE & Guavina guavina \\
Strongylura timucu & PLEURONECTIFORMES \\
SYNGNATHIFORMES & ACHIRIDAE \\
SYNGNATHIDAE & Achirus lineatus \\
Oostethus lienatus & PARALICHTHYIDAE \\
PERCIFORMES & PENTROPOMIDAE \\
Centropomus parallelus & P. orbignyana \\
MUGILIDAE & P. triocellatus \\
Mugil curema & TETRAODONTIFORMES \\
M. liza & TETRAODONTIDAE \\
& Sphoeroides testudineus \\
\hline
\end{tabular}

Embora a introdução de espécies não nativas em ambientes com características insulares, como é o caso de bacias hidrográficas isoladas, seja usualmente danosa e, em casos extremos possa levar a extinção de taxa autóctones (Lodge,
1993), alguns grupos ícticos foram introduzidos na bacia por iniciativas oficiais, como é o caso de $T$. renadlli, $C$. ocellaris e $S$. maxilosus (Barroso, 1989, Bizerril, 1996). 


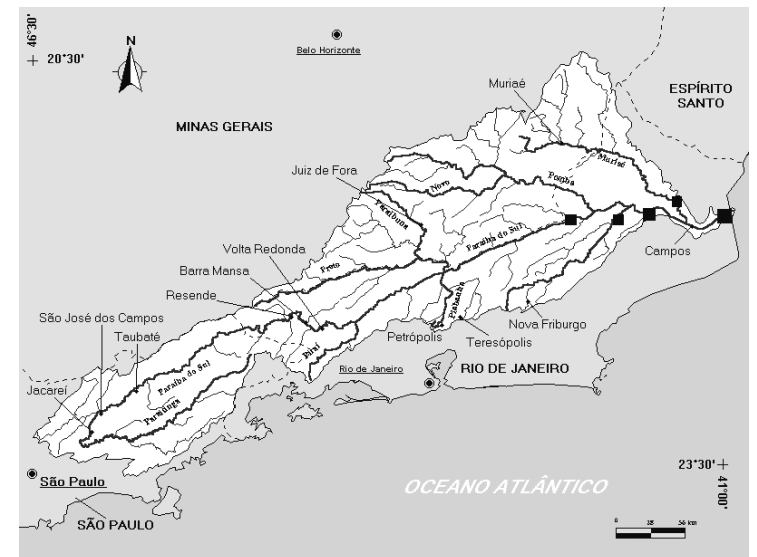

Mugil liza/Anchoviella lepidentostole

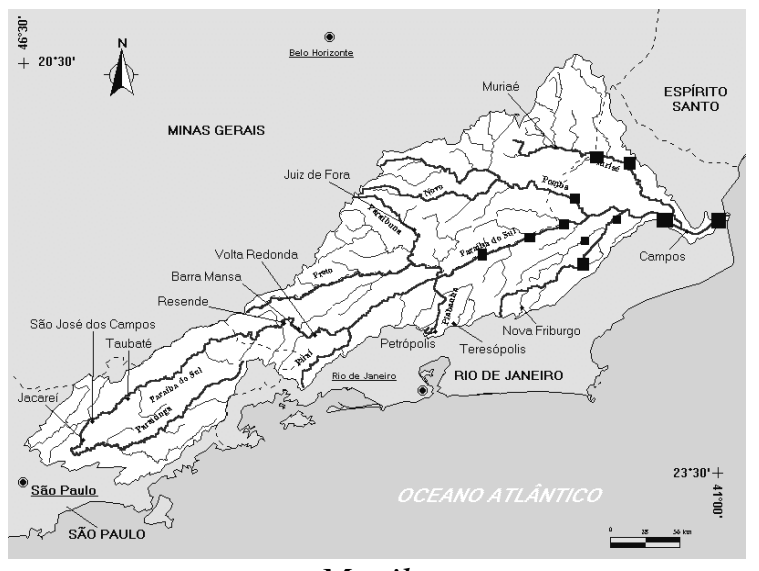

Mugil curema
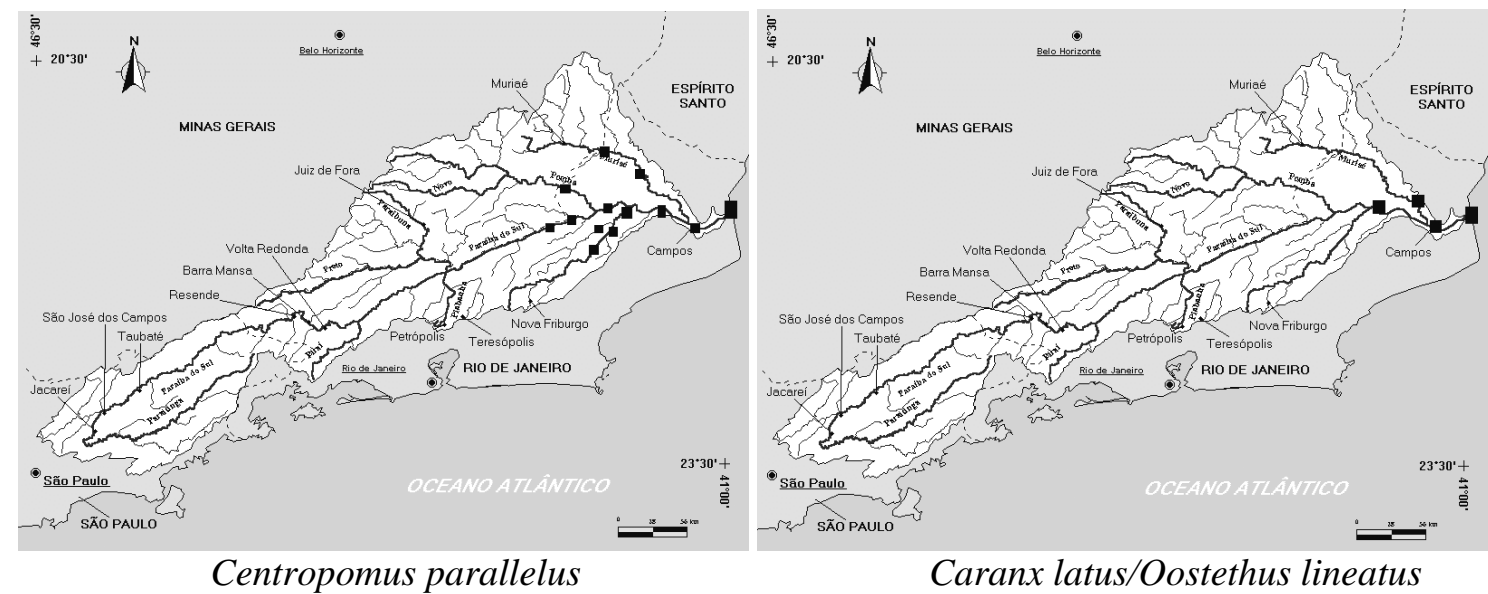

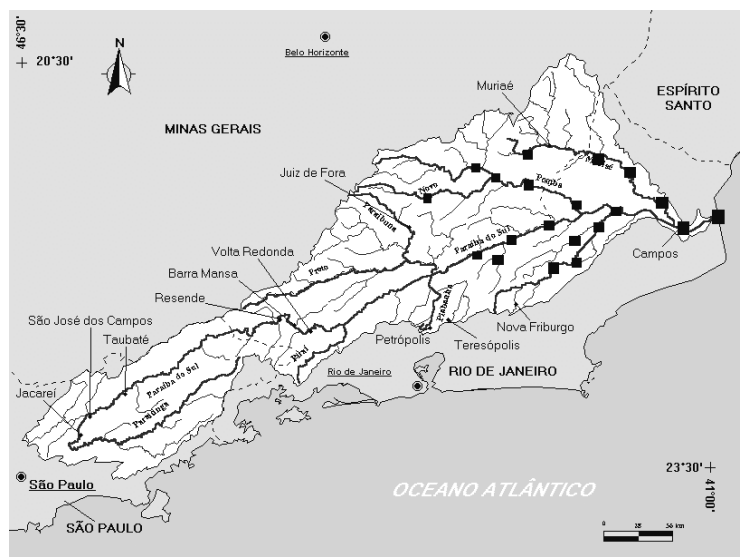

Awaous tajasica

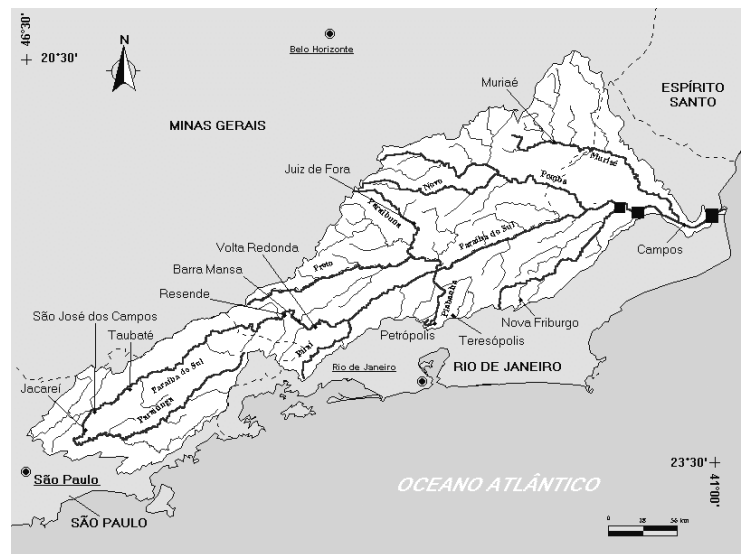

Diapterus rhombeus

Fig.4. Amplitude de distribuição de algumas espécies marinhas eurihalinas na bacia do rio Paraíba do Sul. 
Tabela II. Classificação das espécies introduzidas por domínio geoambiental.

\begin{tabular}{l||c||c||c||c||c||c||c}
\hline \hline \multicolumn{1}{c||}{ CLASSIFICAÇÃO } & DI & DII & DIII & DIV & DV & DVI & DVII \\
\hline \hline Prochilodus scrofa & I & I & I & I & I & I & I \\
Salminus maxilosus & I & I & I & I & I & I & I \\
Colossoma macroponum & A & C & C & A & C & C & A \\
Piaractus mesopotamicus & A & C & C & A & C & C & A \\
Metynnis sp. & A & A & A & C & A & A & A \\
Hyphessobrycon callistus & A & A & A & C & A & A & A \\
Cyprinus carpio & I & I & I & I & I & C & C \\
Ctenopharyngodon idella & A & A & A & A & C & A & A \\
Salmo gairdineri & C & A & A & A & A & A & A \\
Clarias sp. & A & C & C & C & C & C & C \\
Poecilia reticulata & I & I & I & I & I & I & I \\
Xiphophorus helleri & A & A & A & A & C & A & A \\
Aequidens sp. & A & A & A & C & A & A & A \\
Cichla ocellaris & C & I & I & I & I & I & C \\
Tillapia rendalii & I & I & I & I & I & I & I \\
Oreochromis niloticus & C & C & C & C & C & C & C \\
\hline \hline
\end{tabular}

C - Colonizador; I- Invasor; A- Ausente; DI - Domínio dos corpos fluviais; DII - Domínio dos meandros com lagoas marginais, DIII - Domínio de meandros com condicionamento estrutural DIV - Domínio das corredeiras DV - Domínio das ilhas fluviais DVI - Domínio dos depósitos fluviais, DVII - Domínio das lagoas

A semelhança entre a bionomia de espécies nativas e introduzidas é um aspecto que pode conduzir a um processo de exclusão de grupos que possuem menor potencial biótico (Lowe McConnell, 1987, Willians \& Nowack, 1993). Possivelmente este fenômeno, associado às alterações ambientais sofridas pela bacia, poderia explicar a redução descrita por pescadores, dos estoques de Brycon opalinus na bacia e o concomitante aumento na população de Salminus maxilosus. Processo similar se observa com relação ao curimbatá nativo (Prochilodus vimboides), cada vez mais raro, e o seu equivalente introduzido ( $P$. scrofa), mais abundante (Observação pessoal).

Espécies ictiófagas, como Clarias sp. e Cichla ocellaris, se expandirem sua distribuição para áreas que se notabilizam por manterem ictiocenoses com alto grau de endemismo poderão, através de interação predatória, gerar impactos significativos sobre a biodiversidade do Paraíba do Sul, em um processo similar ao observado em outras bacias hidrográficas brasileiras (e.g., Godinho \& Godinho, 1994) e em outros países (Lowe McConnell, 1987, FAO, 1984, Willians \& Nowack, 1993).

A fauna de água doce nativa é formada essencialmente por peixes Otophysi, um arranjo comum às demais bacias da região Neotropical (Lowe McConnell, 1987). Dentre as ordens inventariadas, os Siluriformes foram os que se mostraram maior riqueza de espécies (Fig.5), o que concorda com o padrão descrito por Bizerril (1994) para rios do leste Brasileiro. 


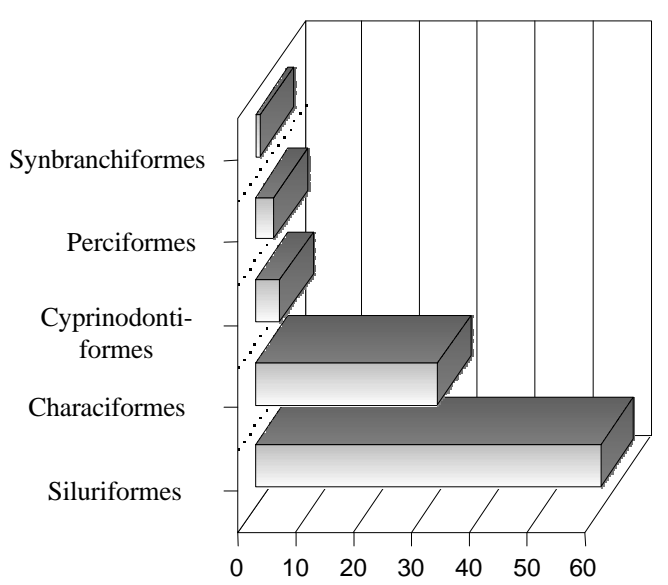

Fig.5. Representatividade (\%) das diferentes ordens

Em sua composição geral verifica-se a ocorrência da maior parte dos gêneros de peixes de água doce registrada nos rios integrados à província ictiogeográfica do sudeste brasileiros dos estados de São Paulo e do Rio de Janeiro, estando ausentes apenas os gêneros Kronichthys, Leptolebias, Rivulus, Pseudotothyris, Trichogenes, Listrura, Pseudocorynopoma, Rachoviscus, Pareiorhaphis, Spintherobolus e Sympsonichthtys. A bacia mantém ainda uma série de taxa que, na província do sudeste brasileiro, the são exclusivos (e.g., Pogonomopoma, Oligobrycon) ou compartilhados com rios do Espirito Santo (e.g. Delturus, Steindachneridion).

Aos grupos supracitados soma-se algumas espécies com representantes em bacias integradas as drenagens do Paraná e do São Francisco (e.g., Pareiorhina, Cheirodon, Rhamdiopsis, Pseudotocinclus, Phallotorhynus) e ausentes em outros sistemas fluviais do leste brasileiro.

A ordem Characiformes reúne 35 espécies (Tabela III), agrupadas em 6 famílias, das quais
Characidae engloba a maior riqueza de taxa.

A distribuição dos Characiformes abrange todos os domínios, o que reflete a grande diversificação morfológica e ecológica do grupo. Logicamente algumas condições mostraram-se mais propícias a determinados grupos.

Assim, áreas de corredeiras, tais como as verificadas em abundância no domínio das corredeiras (D IV) e no alto curso de diversos tributários, mostraram-se particularmente ideais aos Chrenuchiidae e Astyanax scabripinnis, enquanto os remansos, tão característicos dos domínios dos depósitos fluviais (DVI), dos corpos lagunares (DVII) e das ilhas (DV), favorecem de sobremaneira os Erythrinidae, Prochilodontidae, Curimatidae e Anostomidae.

São igualmente comuns nestes locais os peixes pertencentes aos gêneros Astyanax e Cheirodon, bem como Probolodus heterostomus. Peixes como os dos gêneros Brycon e Salminus (exótico) são mais comuns em locais de dinâmica intermediária, tais como margens vegetadas próximas as corredeiras ou nos pontos de conexão fluvial.

Os compartimentos de transporte e de armazenamento dos sistemas fluviais reúnem a maior riqueza de espécies, as quais ocorrem nos microambientes de remansos e na porção marginal dos rios, respectivamente (Fig.6).

As áreas de fonte, por suas condições fortemente limitantes, (e.g., baixa produtividade biológica e alta instabilidade ambiental (Pettis \& Amoros, 1996)), mantêm poucos Characiformes, destacando-se os grupos bentônicos, como os Chrenuchiidae. 
Tabela III. Characiformes nativos da bacia do rio Paraíba do Sul

\begin{tabular}{||l||l||}
\hline ERYTHRINIDAE & CHARACIDAE \\
Hoplias malabaricus & Brycon opalinus \\
Hoplerythrinus unitaeniatus & Brycon sp. \\
PROCHILODONTIDAE & Oligosarcus hepsetus \\
Prochilodus vimboides & Mimagoniates microlepis \\
CURIMATIDAE & Astyanax sp. \\
Cyphocharax gilbert & Astyanax aff bimaculatus \\
ANOSTOMIDAE & Astyanax fasciatus parahybae \\
Leporinus conirostris & A. giton \\
L. copelandi & A. scabripinnis \\
L. mormyrops & A. taeniatus \\
L. cf. thayeri & Bryconamericus sp. \\
CHRENUCHIIDAE & B. tenuis \\
Characidium alipioi & Deuterodon pedri \\
C. interruptum & Hyphessobrycon bifasciatus \\
Characidium sp.1 & H. cf. luetkeni \\
Characidium sp.2 & H. reticulatus \\
Characidium sp.3 & H. duragenys \\
Characidium sp.4 & Cheirodon parahybae \\
C. laroi & Oligobrycon microstomus \\
& Probolodus heterostomus \\
\hline \hline
\end{tabular}

A ordem Siluriformes está representada na bacia por 70 espécies (Tabela IV). Sua maior diversidade se dá nos trechos de transporte e de armazenamento, havendo maior riqueza de taxa nos remansos e nas porções marginais (Fig.7).

Em comparação com os Characiformes, verificase maior riqueza relativa de espécies no alto curso dos rios. Estes locais mostram-se ideais aos Trichomycteridae, além de Neoplecostomus spp. Paraeiorhina spp., Upsilodus victori, Schizolecis guntheri, Corydoras barbatus e Rhamdioglanis transfasciatus, refletindo a própria morfologia dos grupos e o hábito bentônico dos mesmos, que em conjunto reduzem a capacidade de carreamento pelos rios. Nos pontos com águas barrentas e pouco movimentadas predominam Pimelodus maculatus, P. fur, Trachelyopterus striatulus, Glanidium melanopterum, Rhamdia parahybae e Corydoras nattereri. Em áreas intermediárias, tais como aquelas encontradas nos remansos do domínio das ilhas fluviais (DV) e nos encontros de rios, Steindachneridion parahybae coexiste com Imparfinis piperatus, Pimelodella spp. e os iliófagos Loricariichthys sp., Hartia spp., Rineloricaria spp., Hypostomus, Delturus, Pogonopomoides, Rinelepis. 


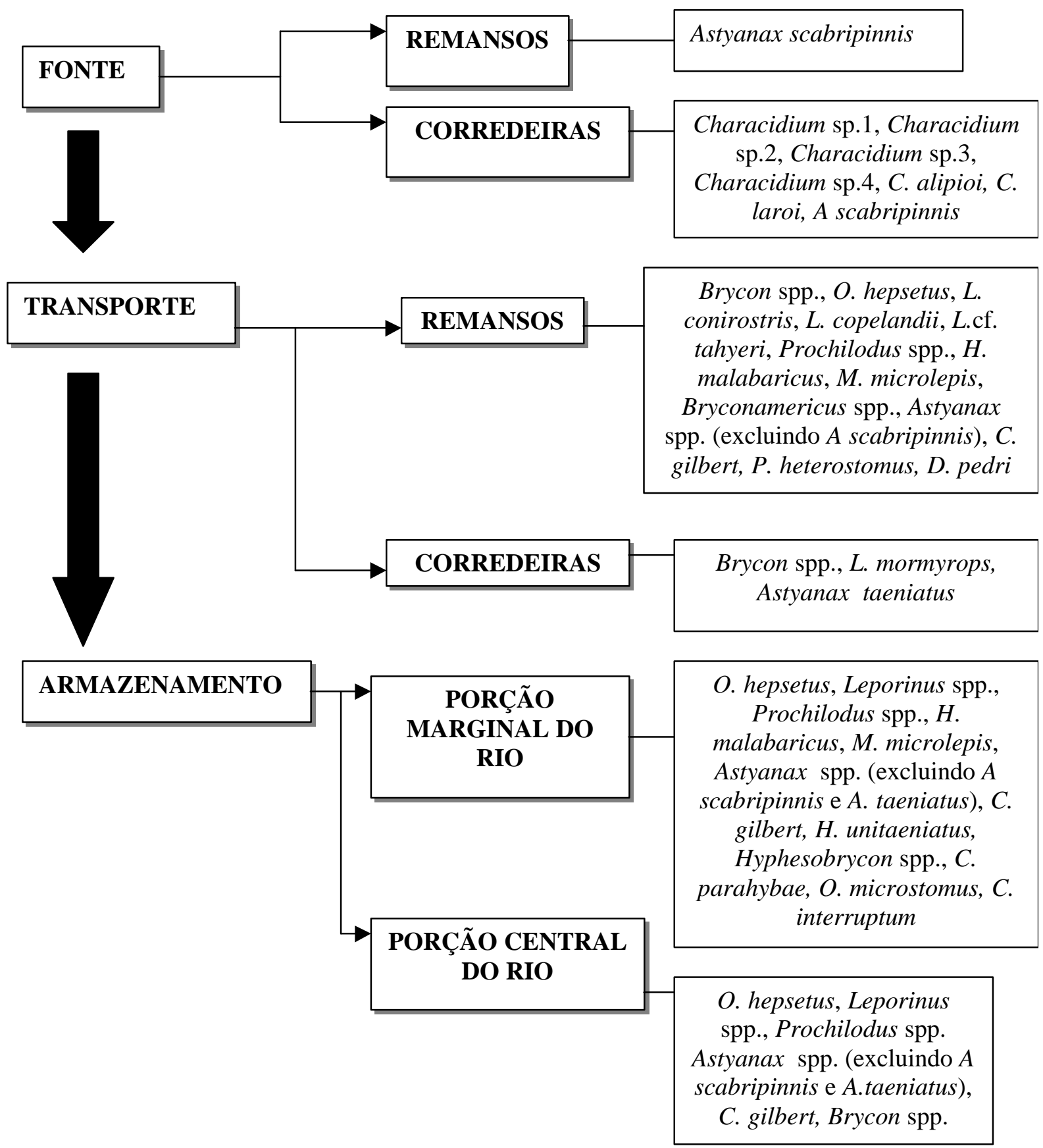

Fig.6. Distribuição dos Characiformes na bacia do rio Paraíba do Sul.

Os Cyprinodontiformes estão representados pelas famílias Poeciliidae [Poecilia vivipara Phalloceros caudimaculatus, Phallopthychus januarius, Phallotorhynus fasciolatus)] e Anablepidae [Jenynsia multidentata)]. São comuns em remansos, ocorrendo inclusive em áreas com forte influência salina, como se observa para as espécies nativas dos gêneros Poecilia, Phallopthychus e Jenynsia. 
Tabela IV. Siluriformes nativos da bacia do rio Paraíba do Sul

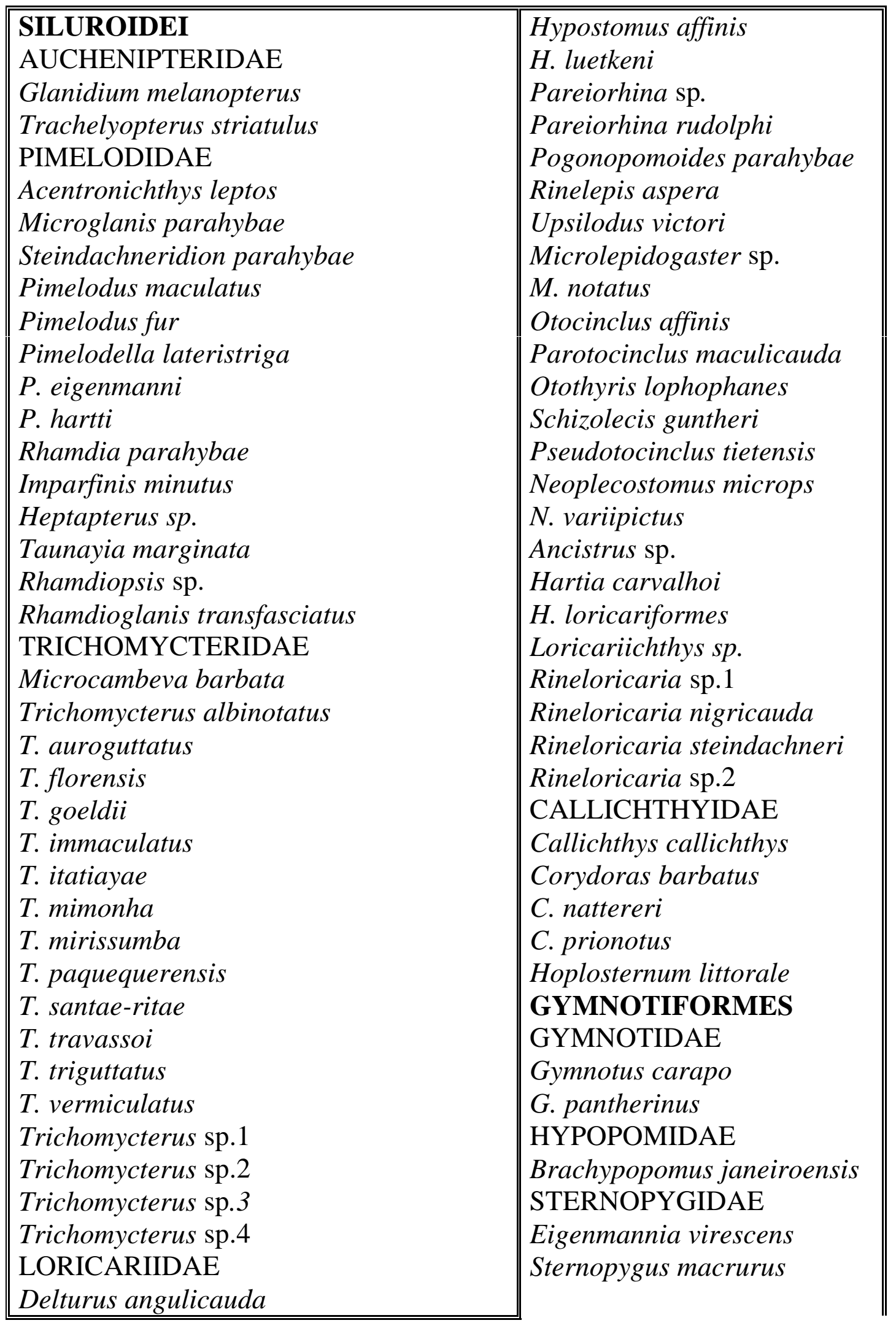


A ordem como um todo ocorre em todos os domínios considerados. Jenynsia e Phallopthychus encontram-se apenas no domínio 7, enquanto Phallotorynus fasciolatus é restrito aos domínios 1 e 2. Poecilia vivipara se faz presente nos domínios 4, 5, 6 e 7 .

A ordem Synbranchiformes possui, na bacia, apenas uma espécie (Synbranchus marmoratus), que ocorre em todos os domínios reconhecidos.

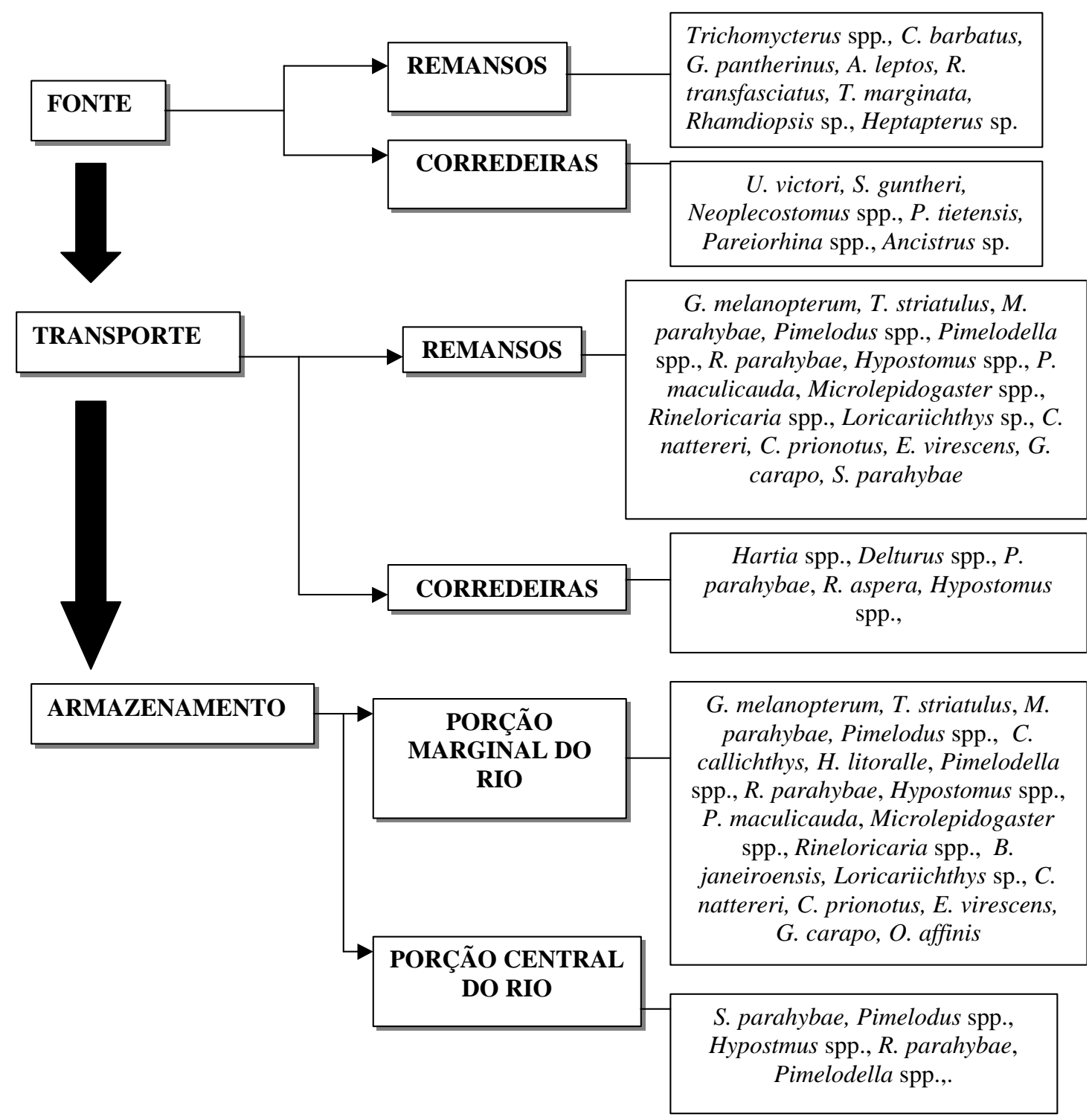

Fig.7. Distribuição característica dos Siluriformes por ambientes na bacia do rio Paraíba do Sul.

Espécies dulcícolas de Perciformes ocorrem em Cichlidae [Geophagus brasiliensis, Cichlasoma facetum, Crenicichla lacustris)], Gobiidae (Awaous tajasica) e Scianidae (Pachypops adspersus). De um modo geral a maior densidade e diversidade de Perciformes se dá em áreas remansosas, especialmente nos setores de armazenamento e de transporte. C. lacustris e A. tajasica, contudo, são freqüentemente registrados em trechos dos rios nos quais há maior circulação de água, ocasionalmente ocultando-se entre rochas nas piscinas formadas abaixo de corredeiras.

Peixes de água doce, por exibirem capacidade de dispersão restrita ao canal fluvial, constituem 
um grupo faunístico particularmente indicado para estudos biogeográficos que objetivem reconhecer e remontar paleocomunicações entre ambientes atualmente isolados. Assim sendo, a presença de espécies comuns em sistemas fluviais distintos e não comunicantes pode ser interpretada como uma indicação da ocorrência de processos de dispersão e vicariância entre as unidades geográficas envolvidas (Mayden, 1992).

No caso específico da bacia do rio Paraíba do Sul, a análise comparativa da ictiofauna presente neste sistema com a ocorrente nas demais bacias do sudeste permitiu identificar áreas que representam um antigo continunn de ambientes, ilustrando paleo-comunicações derivadas de interconexão fluvial ao nível da plataforma continental ou de erosão remontante de cabeceiras.

Dentro do arranjo ictiofaunístico da bacia do rio Paraíba do Sul, podem ser reconhecidas 4 grandes unidades de afinidades ictiogeográficas.

A unidade 1 engloba o trecho médio inferior e o baixo Paraíba do Sul, caracterizando-se pela alta similaridade com sistemas fluviais situados entre o rio Una e as bacias das lagoas de Cima e Feia (ao sul) e entre o rio Itabapoana e Beneventes, ES (ao norte).

A unidade 2 (i.e., alto curso do rio Santo Antônio e São Lourenço, na bacia do rio Grande/Dois Rios, RJ) compartilha diversas espécies, tais como Corydoras barbatus, Acentronichthys leptos, Schizolecis guntheri e Rhamdioglanis frenatus, com o alto curso do rio Macacu, um rio que drena diretamente à baia de Guanabara. Este aspecto ilustra a captura fluvial deste sistema pela bacia do rio Grande/Dois Rios.

De forma similar, a unidade 3, localizada no alto rio Paraibuna, guarda algumas espécies ou gêneros comuns com o alto rio Paraná (alto rio Grande, MG), sugerindo um segundo evento de captura fluvial.

Capturas fluviais envolvendo o rio Paraíba do Sul/Tietê, um fenômeno que se encontra bem documentado na literatura, tanto sob o ponto de vista geológico (Ab'Saber \& Bernardes, 1958) como quanto a aspectos bióticos (cf. Langeani, 1989), caracterizam a quarta unidade que se situa entre a nascente do rio e a localidade de Jacareí (inclusive).

Em sua composição geral, a ictiofauna do rio Paraíba do Sul revela que este sistema foi formado pela interseção de sua bacia original com rios atualmente associados às bacias costeiras, do alto Paraná e do Tietê, todas estas com divisores de águas atualmente separando-as do complexo do Paraíba.

Adotando a aproximação biogeográfica na qual a bacia hidrográfica se torna a unidade de análise observa-se que, como reflexo das características fisiográficas atuais e da história de formação de cada um dos domínios ambientais, verifica-se uma distribuição não homogênea das espécies (Fig. 8), havendo uma aumento na diversidade biológica ao longo do gradiente lótico, seguindo um padrão esperado dentro do princípio de continuidade de rios (Vannote et al., 1980).

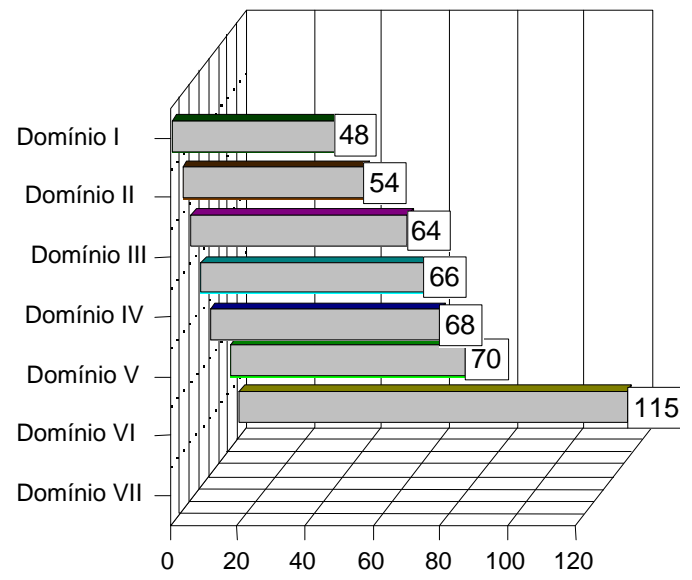

Fig.8. Número de espécies por domínio geoambiental

Comparando as ictiocenoses de cada domínio no que se refere à sua composição qualitativa, obtêm-se o arranjo apresentado na Fig.9, onde se observa a existência de 5 domínios ictiogeográficos (I- Domínio das Serras; II Domínio dos Meandros; III - Domínio dos Meandros Estruturais + Domínio das Corredeiras; IV - Domínio das Ilhas + Domínio dos Depósitos Fluviais e V - Domínio dos Corpos Lagunares), os quais podem, por suas semelhanças estruturais, ser trabalhados, em 
programas ambientais específicos, como unidades naturais.

Foi obervado que algumas regiões da bacia do rio Paraíba do Sul (Tabela V) notabilizam-se por reunirem conjuntos únicos de espécies. Tais unidades (i.e., zonas de endemismo) atuam, em casos como Z1, Z2 e Z3, como refúgios faunísticos para espécies que apresentavam, em períodos geológicos anteriores, uma distribuição geográfica mais ampla, tanto nos limites da bacia do rio Paraíba do Sul, como em outras unidades biogeográficas.

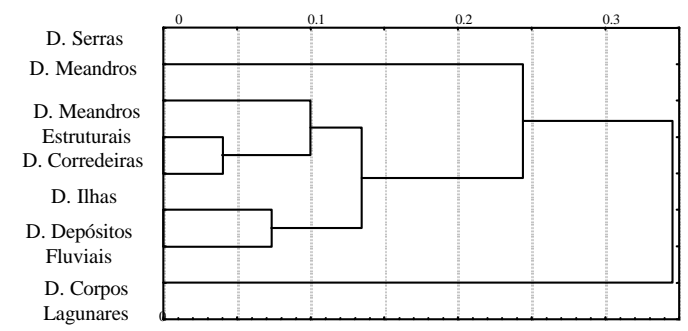

Fig. 9. Agrupamento por UPGMA dos escores de dissimilaridade (Percentual de discordância) obtidos na comparação das ictiocenoses dos diferentes domínio geoambientais.
A bacia do rio Paraíba do Sul exibe, no presente, expressiva alteração na qualidade da água (Mello, 1997). Embora em pontos localizados se evidenciem concentrações de poluentes diferenciados, a bacia como um todo se notabiliza por exibir altas concentrações de sólidos (em especial sedimentos) em suspensão (Mello, 1997), derivados principalmente dos processos erosivos que se estabeleceram nas margens fortemente desmatadas.

A transformação de grandes rios de águas límpidas em sistemas com alta carga de sedimentos em suspensão é um processo com rebatimentos em diversos grupos da fauna aquática, tendo sido apontado como a causa de extinção de diversas espécies de peixes na região neártica (Willians \& Nowack, 1993).

A ausência de dados acerca da ictiofauna presente no rio Paraíba do Sul em períodos anteriores aos grandes desmatamentos impede avaliar a magnitude deste processo impactante sobre a fauna local, ou ainda identificar a ocorrência de extinções derivadas do mesmo.

Tabela V. Zonas de endemismo da bacia do rio Paraíba do Sul.

\begin{tabular}{|c|c|}
\hline ZONAS DE ENDEMISMO & DESCRIÇÃO \\
\hline $\begin{array}{l}\mathrm{Z} 1 \text { - Alto rio Paraíba e sistemas tributários } \\
\text { associados }\end{array}$ & 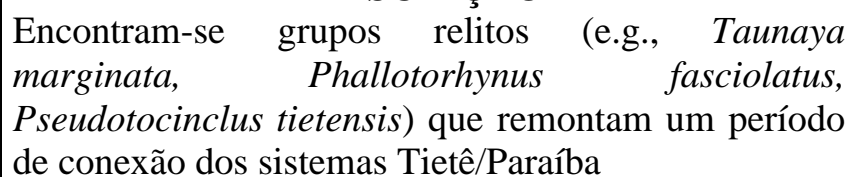 \\
\hline $\begin{array}{l}\mathrm{Z2} \text { - Alto rio Preto e sistemas integrados ao } \\
\text { Parque Nacional de Itatiaia }\end{array}$ & $\begin{array}{l}\text { Se registram diversos grupos exclusivos do local, } \\
\text { como é o caso de Characidium lauroi, Pareiorhina } \\
\text { rudolphi e Trichomycterus itatyae, além de diversas } \\
\text { espécies de Trichomycterus descritas apenas } \\
\text { recentemente (Costa, 1992) }\end{array}$ \\
\hline $\begin{array}{l}\text { Z3 - Alto rio Paraibuna e bacias associadas } \\
\text { Z4 - Alto rio Grande (rio São Lourenço) }\end{array}$ & $\begin{array}{l}\text { Endemismos de Trichomycterus e Rhamdiopsis } \\
\text { Ictiofauna guarda uma série de elementos } \\
\text { característicos dos rios que correm pela vertente leste } \\
\text { da Serra do Mar (e.g., Corydoras barbatus, } \\
\text { Rhamdioglanis frenatus, Schizolecis guntheri), } \\
\text { representando uma área de captura fluvial }\end{array}$ \\
\hline & Mantém espécies exclusivas, como T. paquequerensis \\
\hline
\end{tabular}

Pode-se, contudo, inferir que esta alteração ambiental mostra-se mais atuante sobre grupos que dependem da orientação visual para a captura de presas, podendo ser, portanto, uma das causas múltiplas que vêm contribuíndo para o declínio das espécies de Brycon na bacia.

Embora delimitar a ocorrência de perdas bióticas 
pretéritas na bacia seja atualmente inviável, é possível observar a ocorrência de um processo de extinções bem representado nos seguintes setores:

Rio Paraíba do Sul entre Resende e Volta Redonda, onde o lançamento de efluentes domésticos e industriais, se reflete na contaminação do pescado e na eliminação de espécies mais sensíveis (FEEMA, 1979; Barroso, 1989);

Rio Paraíbuna, a jusante da cidade de Juiz de Fora, onde se repetem os processos identificados na primeira região;

Rio Piabanha, sistema que, devido ao lançamento de efluentes, encontra-se desprovido de fauna na maior parte de sua extensão. Nesta bacia, a rede de drenagem, que ainda guarda alguns sistemas bem conservados, atua como último refúgio faunístico para as espécies locais. Melhores condições ambientais começam a ser identificadas apenas após o encontro do rio Piabanha com o rio Preto;

Rio Grande, entre Conselheiro Paulino e Bom Jardim, onde o efeito do lançamento de efluentes é magnificado pela reduzida vazão relativa do rio. $\mathrm{O}$ efeito da poluição encontra-se refletido tanto nas perdas bióticas como na ocorrência, no trecho em enfoque, de deformidades e lesões em Hypostomus affinis e em Rhamdia parahybae (Bizerril, 1997a);

Rio Muriaé, na cidade de Muriaé, onde o lançamento de efluentes gerados na cidade em um trecho de baixa dinâmica de circulação se reflete no aumento significativo de coliformes, DBO, DQO e extinção local de espécies de peixes que, até este ponto, se distribuíam de forma homogênea e contínua (Bizerril, 1997b).

O processo de extinção das espécies se dá em escalas de tempo distintas, podendo ser rapidamente evidenciado quando do desaparecimento de determinados taxa ou da redução progressiva nos estoques de sua população, como se verifica na bacia com grupos como Steindachneridion parahybae e Brycon spp. Em outras situações, de detecção menos simples, a fragmentação no habitat conduz à gradual redução na diversidade genética das populações afetadas, gerando, a longo ou médio prazo, seu desaparecimento (Avise \& Hamrick, 1996).

O último evento possivelmente vem se estabelecendo em áreas como o rio Piabanha e médio rio Grande, por exemplo, ambientes nos quais as alterações na qualidade da água vem gerando barreiras para o intercâmbio gênico entre as populações de diferentes espécies ícticas confinadas rede de tributários com melhores níveis de integridade ambiental, a maioria dos quais dotados de pequenas dimensões e reduzidas ordens (sensu Strhaler, 1952 apud Petts \& Amoros, 1997), o que produz um arranjo de baixa capacidade suporte.

\section{AGRADECIMENTOS}

À equipe envolvida no Projeto Paraíba do Sul pelo apoio técnico. Todos os mapas foram desenhados sobre bases elaboradas pela Cooperação Brasil/França.

\section{REFERÊNCIAS BIBLIOGRÁFICAS}

Ab'Saber, A.N. \& Bernardes, N (1958), Vale do Paraíba, serra da Mantiqueira e arredores de São Paulo. Conselho Nacional de Geografia, Rio de Janeiro.

Andreata, J.V., Saad, A.M. Bizerril, C.R.S.F. \& Bockmann, F.A. (1990), Alguns aspectos da ecologia das espécies de peixes da Laguna da Tijuca, Período de março de 1987 a fevereiro de 1989. Acta Biol. Leopold, 12, 247-268.

Araújo, J.R.S. (1983), Projeto de biodetecção de tóxicos em sistemas fluviais de utilização em captação de água para sistemas públicos de abastecimento. FEEMA, Rio de Janeiro.

Araújo, G.F. (1996), Composição e estrutura da comunidade de peixes do médio e baixo rio Paraíba do Sul, RJ. Rev. Brasil. Biol., 56, 111-126.

Araújo, G.F. (1985), Levantamento preliminar dos organismos aquáticos do rio Paraíba do Sul - RJ. Relatório final. Convênio FINEP/Posto de Aquicultura/UFRRJ, Rio de Janeiro. 
Avise, J.C. \& Hamrick, J.L. (1996), Conservation genetics - Case histories from nature. Chapman \& Hall, New York.

Barroso, L.V. (1989), Diagnóstico ambiental para a pesca de águas interiores no Estado do Rio de Janeiro. IBAMA, Rio de Janeiro.

Bizerril, C.R.S.F. (1994), Análise taxonômica e biogeográfica da ictiofauna de água doce do leste brasileiro. Acta Biol. Leopoldensia, 16, 51-80.

Bizerril, C.R.S.F. (1995a), Estrutura quantitativa de comunidades de peixes em um rio costeiro do sudeste brasileiro. Acta Biol. Leopoldensia, 17, 57-80.

Bizerril, C.R.S.F. (1995b), Descrição de uma nova espécie de Neoplecostomus (Loricariidae, Neoplecostominae), com uma sinópse da composição taxonômica dos Loricariidae no leste brasileiro. Arq. Biol. Tecnol., 38, 693-704.

Bizerril, C.R.S.F. (1996), Ictiofauna da bacia do rio Paraíba do Sul - Diversidade biológica, distriubição geográfica $e$ estratégias de conservação - Relatório Final. Projeto Paraíba do Sul/Cooperação BrasilFrança, Rio de Janeiro.

Bizerril, C.R.S.F. (1997a), Programa de monitoramento ds condições bióticas - Rio Grande/Dois Rios (Relatório de andamento). Projeto Paraíba do Sul/Cooperação BrasilFrança, Rio de Janeiro.

Bizerril, C.R.S.F. (1997b), Programa de monitoramento ds condições bióticas - Rio Muriaé (Relatório de andamento). Projeto Paraíba do Sul/Cooperação Brasil-França, Rio de Janeiro.

Bizerril, C.R.S.F., Peres-Neto, P.R. \& Bockmann, F.A. (1996), Morphological and phylogenetic diversification of freshwater fishes from eastern Brazilian rivers. Resumo apresentado no $76^{\text {th }}$ Annual Meeting of American Ichthyologists and Herpetologists, p. 198, 13-19 de Junho. New Orleans.

Bockmann, F.A., Castro, R.M.C. \& Bizerril, C.R.S.F. (1996), Sistemática e biogeografia dos bagres do gênero Rhamdiopsis Haseman, 1911 (Siluriformes, Pimelodidae). Resumo apresentado no XXI Congresso Brasileiro de Zoologia, 5-9 de Fevereiro, Porto Alegre.

Britski, H.E. (1972), Peixes de água doce do Estado de São Paulo. In- Poluição $e$ pisicultura, ed. Comissão Interestadual da Bacia do Paraná-Uruguai. USP/Instituto de
Pesca da C.P.R.N, São Paulo, pp. 79-108.

Caramaschi, E.P. \& Caramaschi, U. (1991), Taxonomic status of the trichomycterid catfish Trichomycterus itatiayae. Copeia, 1991, 222-224.

Costa, G. (1994), Caracterização histórica, geomorfológica e hidráulica do estuário do rio Paraíba do Sul. Dissertação de Mestrado, Universidade Federal do Rio de Janeiro, Rio de Janeiro, Brasil.

Costa, W.J.E.M. (1992) Description de huit nouvelle espèces du genre Trichomycterus (Siluriformes: Trichomycteridae) du Brésil oriental. Rev. fr. Aquariol., 18, 101-110.

Drumond, J.A. (1988), Crescimento populacional e conservação da natureza: considerações sobre o caso brasileiro. Bol. $F B C N, \mathbf{2 3}, 7-12$.

Engevix/UFRJ (1991), Levantamento da ictiofauna do rio Paraíba do Sul e ciclo reprodutivo das principais espécies, no trecho compreendido entre Três Rios e Campos (4 volumes). Furnas Centrais Elétricas, Rio de Janeiro.

Espirito-Santo, C.E., Oyakawa, O.T.. Manna de Deus, J.R. \& Camargo, F.A. (1997), Manual de peixes da Serra da Mantiqueira, vale do Paraíba, SP com ocorrências na Fazende São sebastião do Ribeirão Grande, Pindamonhangaba. Resumo apresentado no XII Encontro Brasileiro de Ictiologia, 24-28 de Fevereiro, São Paulo.

FAO (1984), Documents presented at the symposium on stock enhancement in the management of freshwater fish. Vol. 2 Introductions and transplantations. FAO, Budapest.

FEEMA (1979), Diagnóstico ambiental do Estado do Rio de Janeiro. FEEMSA, Rio de Janeiro.

Godinho, H.P. \& Godinho, A.L. (1994), Ecology and conservation of fish in southeastern brazilian river basins submitted to hydroelectric impoundments. Acta Limnologia Brasiliensia, 5, p.187-198.

Langeani, F. (1989), Ictiofauna do alto curso do rio Tietê (SP): Taxonomia.. Dissertação de Mestrado, Universidade de São Paulo, São Paulo, Brasil.

Langeani, F. (1990), Revisão do gênero Neoplecostomus Eigenmann \& Eigenmann, 1888, com a descrição de quatro novas espécies do sudeste brasileiro (Ostariophysi, 
Siluriformes, Loricariidae). Comun. Mus. Ciênc. PUCRS, sér. Zool., 3, 3-31.

Lodge, D.M. (1993), Species invasions and deletions: Community effects and responses to climate and habitat change. In- Biotic interactions and global change ed. Kareiva, P.M., Kingsolver. J.G. \& Huey, R. Sinauer Publish, Massachusetts, pp. 367-387.

Lowe McConnell, R.H. (1987), Ecological studies in tropical fish communities. Cambridge University Press, Cambridge.

Mayden, R.L. (1992), Systematics, historical ecology \& North American freshwater fishes. Stanford University Press, Stanford.

Mazzoni, R, Caramaschi , U. \& Weber, C. (1993), Taxonomical revision of the species of Hypostomus Lacépède, 1803 (Siluriformes, Loricariidae) from the lower Paraíba do Sul, State of Rio de Janeiro, Brazil. Revue Suisse de Zoologie, 101, 3-18.

Mello, C.S.B. (1997), Classificação das estações de qualidade de água da bacia do rio Paraíba do Sul em função da análise de componentes principais. Relatório Técnico, Agência Técnica da Bacia do Rio Paraíba do Sul, Rio de Janeiro.

Miranda- Ribeiro, A. (1902), Oito espécies do rio Pomba. Bol. Soc. Nac. Agric., 8: não paginado.

Nomura, Y. (1978), Ictiologia e pisicultura. Livraria Nobel, São Paulo.
Nunann, G.W., Cardoso L.W., \& Bandeira, W.D. (1983), Levantamento da ictiofauna do rio Paraíba do Sul. Trecho Represa do Funil Cidade de Barra do Piraí, Estado do Rio de Janeiro. Resumo apresentado no $\mathrm{X}$ Congresso Brasileiro de Zoologia, 10-14 de março, Belo Horizonte.

Petts, G.E. \& Amoros, C. (1997), Fluvial hydrosystems. Chapman \& Hall, London.

Suppa, A. \& Buckup, P.A. (1997), As espécies de Rineloricaria (Siluriformes, Loricariidae) na bacia do rio Paraíba do Sul. Resumo apresentado no XII Encontro Brasileiro de Ictiologia, 24-28 de Fevereiro, São Paulo.

Vannote, R.L., Mishall, G.W., Cummins, K.W. \& Cushing, C.E. (1980), The river continunn concept. Can. J. Fish. Aquat. Sci., 37, 130137.

Willians, J.D. \& Nowack, R.M. (1993), Vanishing species in our own backyard: Extinct fish and wildlife of the United States and Canada. In - The last extinction, ed. Kaufman, L. \& Mallory, K.. Massachusetts Institute of Technology, Massachucetts, pp. 115-148. 